\title{
Après le Bilan de santé de la PAC. Quelle politique agricole commune après 2013 ?
}

\section{Franco Sotte et Emilio Chiodo}

\section{(2) OpenEdition}

Édition électronique

URL : http://journals.openedition.org/economierurale/2583

DOI : 10.4000/economierurale.2583

ISSN : 2105-2581

Éditeur

Société Française d'Économie Rurale (SFER)

Édition imprimée

Date de publication : 5 avril 2010

Pagination : 69-76

ISSN : 0013-0559

\section{Référence électronique}

Franco Sotte et Emilio Chiodo, «Après le Bilan de santé de la PAC. Quelle politique agricole commune après 2013 ? », Économie rurale [En ligne], 316 | mars-avril 2010, mis en ligne le 05 avril 2012, consulté le 22 avril 2019. URL : http://journals.openedition.org/economierurale/2583 ; DOI : 10.4000/ economierurale. 2583

(C) Tous droits réservés 


\section{- Après le Bilan de santé de la PAC Quelle politique agricole commune après 2013 ?}

Franco SOTTE

Università Politecnica delle Marche, Ancona, Italie
Emilio CHIODO

Università degli Studi di Teramo, Italie

Après le bilan de santé de la PAC, il convient de s'interroger sur le devenir de la politique agricole commune après 2013. Le document conclusif du Bilan de santé dresse un certain nombre de préconisations. Les auteurs, Franco Sotte et Emilio Chiodo, se sont penchés sur la particularité de ces résolutions. Confèrent-elles à la PAC un caractère pertinent et durable ? Quelle sera la dotation financière de l'agriculture dans ces ajustements ? (NDLR)

A près un laborieux processus s'étant étalé sur un an, le Bilan de santé de la PAC se terminait le 20 novembre 2008. D'après le commissaire à l'Agriculture, Mme Fischer Böel, il s'agissait «...d'un ajustement des réformes de 2003 et d'une contribution au débat sur les priorités à venir dans le domaine de l'agriculture » (Commission européenne, 2007)'.

Le but de ce débat n'est pas d'analyser les détails techniques des décisions complexes contenues dans le document conclusif du Bilan de santé. Il s'agit plutôt de mener une réflexion poursuivant deux objectifs. Le premier est d'examiner la manière dont le compromis définitif du Bilan de santé achève effectivement la réforme Fischler. Le second est d'en évaluer les conclusions. Leurs caractéristiques apportent-elles à la PAC crédibilité et durabilité, même après 2013 ? La PAC est-elle assurée d'une dotation financière satisfaisante lors du réexamen du budget, actuellement en cours ? Les décisions à venir (d'ici 2012, selon les prévisions) donnent-elles une plus grande visibilité dans les perspectives financières de la PAC ?

1. Commission européenne (2007). Agriculture: bilan de santé pour rationaliser la politique agricole commune et relever de nouveaux défis. Bruxelles, 20 novembre 2007, IP/07/1720.

\section{Le Bilan de santé a-t-il achevé la réforme Fischler?}

Pour répondre à cette première question, il convient de parcourir à nouveau les points de la réforme de 2003. Bien qu' ayant commencé comme simple « révision à moyen terme » de l'Agenda 2000, la réforme Fischler a été l'objet de commentaires très favorables au niveau des solutions adoptées devant assurer un équilibre global. D'une part, on réalisait une fois pour toutes le découplage entre paiements et productions agricoles (sauf cas résiduels de couplage à éliminer avec le temps) ; d'autre part, avec le régime de paiement unique (RPU), on trouvait une solution ne pénalisant pas ceux qui, en suivant les indications de la PAC jusque-là en vigueur, avaient investi dans les secteurs productifs mais dont les prix n'auraient plus été soutenus.

Le jugement positif à porter au crédit de la réforme Fischler était donc lié à sa capacité de libérer, sans traumatismes, l'agriculture solidaire d'une politique qui :

- construisait, en altérant artificiellement les signaux du marché, des positions de rente empêchant l'exercice de l'entreprise et le renouvellement générationnel ;

- provoquait des effets de distribution altérés, en favorisant un nombre limité d'entreprises et de territoires plus dotés, au 
détriment de ceux qui se caractérisaient par un retard structurel ;

- posait des obstacles au processus d'intégration européenne (en particulier au détriment des nouveaux États membres);

- opposait l'Europe au reste du monde, dans un contexte international d'élargissement et d'ouverture des marchés.

Il est évident que la solution adoptée avait, et il ne pouvait pas en être autrement, un caractère transitoire : le résultat était à la fois un point d'arrivée et un point de départ dans le parcours d'identification d'une stratégie pour le secteur primaire et pour les territoires ruraux, donc pour l'ensemble de la politique agricole.

Dès les premières propositions du Bilan de santé, on ne peut pas dire qu'elles aient tenu compte de la nature transitoire qui était à la base de la réforme Fischler. Au contraire, on peut observer comment le document d'ouverture a essentiellement ciblé les thématiques laissées ouvertes par cette réforme, en particulier sur le premier pilier, en n'abordant le second que de manière indirecte et désorganisée.

En effets, malgré les affirmations de Mme Fischer Böel : «Rural development must form part of the so-called "CAP Health Check" » (Fischer Böel, 2007a) et «Rural development policy ... this is where music is playing » (Fischer Böel, 2007b)2 , il n'y a, dans le document, aucune analyse du second pilier qui était, lui-aussi, dans une phase particulièrement critique, caractérisée par une mise en place lente et problématique.

Plus de trois ans après le règlement 1698/2005 et à plus de deux ans de la mise en place de la politique de développement rural (2007-2013), on attendait du Bilan de

2. Fischer Böel M. (2007a). Rural development in the European Union, International LEADER conference. Gyöngyös, Hungary, 25 october. Fischer Böel M. (2007b). Future Policies for Rural Europe and Beyond - Delivering Sustainable Land Management in a Changing Europe. 19 septembre. santé une vérification de l'état de santé même du second pilier (s'ajoutant au premier pilier), ce qui aurait permis de recenser les réalisations et apporter les corrections propices à la structure normative et procédurale. Les lignes directrices auraient été définies pour affronter les nouvelles tâches transférées du premier au second pilier et, finalement, auraient confirmé l'engagement de la Commission envers la politique de développement rural ${ }^{3}$.

De plus, la connexion entre le premier et le second pilier ressort bien dans le Bilan de santé quand il est affirmé la nécessité de renforcer le second pilier avec une augmentation de la modulation pour affronter les « nouveaux défis » de l'agriculture.

En ce qui concerne la modulation, la proposition initiale d'augmentation (de $5 \%$ à $13 \%$ en régime) pouvait déjà être considérée comme relativement modeste, comparée au $20 \%$ proposés à l'origine par Fischler en 2002 (s'il s'agissait d'achever la réforme, pourquoi viser plus bas que cinq ans auparavant?).

Quant aux «nouveaux défis », (le changement climatique, les énergies renouvelables, la gestion de l'eau, la biodiversité),

3. La nécessité d'un vérification stratégique ressort de plusieurs éléments, comme la difficulté à mettre en marche les Programmes de développement rural ou le fait que les régions se montrent plus rapides dans l'activation des mesures les plus faciles à administrer, en négligeant ou en différant les mesures les plus complexes, mais en même temps plus innovantes et qualifiantes. En général, il faut le souligner, le risque est que l'on s'intéresse plus à dépenser n'importe comment toutes les ressources mises à disposition par Bruxelles, plutôt qu'à bien les dépenser, de façon sélective et avec une vision stratégique. Ainsi, comme l'a déclaré dans le passé la Cour des comptes européenne ( $c f$. Rural Development Investments: do they effectively address the problems of rural areas? 2007, Special Report N. 7/2006, European Court of Auditors, 2006), il y a le risque que dans la politique de développement rural prédomine une dangereuse logique de distribution peu attentive à la sélection, à la concentration et à la finalisation des interventions, et donc à l'efficience et à l'efficacité de la politique toute entière. 
ils se sont limités à la rédaction d'une liste, alors que ces thèmes auraient pu être utilisés pour lancer une proposition crédible dans la future politique agricole européenne.

Ainsi, pour ce qui concerne la définition de nouveaux objectifs de la politique de développement rural d'une part et, d'autre part, la prescription de changements souhaitables et véritablement importants, l'engagement de la réforme est jugé insuffisant par rapport à l'engagement du transfert de ressources modulé du premier au second pilier (ce qui était déjà, dans la proposition initiale, inférieures aux attentes, de notre point de vue).

Pour la politique de développement rural, de nouvelles tâches s'accumulent. À titre d'exemple, les renvois au second pilier s'ajoutent en raison, notamment, des problèmes qui se posent avec l'achèvement de la réforme du premier pilier. En matière de découplage, il faut mentionner :

- la question des producteurs laitiers des régions sensibles, suite à la suppression progressive des quotas laitiers ;

- les effets sur l'environnement de la suppression des jachères obligatoires, etc.

En revanche, le thème de la gestion des risques, qui devient crucial avec la réduction des mesures d'intervention sur les marchés, semble avoir été éliminé des tâches transférées au second pilier.

En ce qui concerne le premier pilier, les contenus de la proposition initiale étaient explicitement orientés vers l'achèvement de la réforme de 2003 : découplage complet, régionalisation obligatoire, limitations maximales et minimales aux droits de paiement unique, fin des mesures de contrôle de l'offre, renforcement de l'ex article 69, simplification de la conditionnalité.

Par rapport à ces points, on peut conclure que Mme Fischer Böel a réussi à défendre assez bien sa proposition. Elle a obtenu pratiquement satisfaction pour chaque point des négociations. Cependant, alors que sur les quotas laitiers, l'abolition des mesures de marché, le découplage complété et la modulation, elle a relativement peu cédé, sur d'autres points tels que la régionalisation (qui constituait un des aspects qualifiants de la proposition et qui de mesure obligatoire devenait facultative), tout va demeurer en l'état. Sur d'autres points encore, comme le nouvel article 68 ou les mesures d'accompagnement dans le secteur laitier, le compromis final propose des solutions ambiguës, ou des solutions dont les effets sont peu importants comme, par exemple, le prélèvement supplémentaire de $4 \%$ pour les paiements dépassant les 300000 euros, et dans lequel l'introduction du principe d'un maximal n'entraîne que des conséquences mineures.

Finalement, en encourageant une confrontation low profile, interne aux intérêts agricoles, et en catalysant l'attention sur le premier pilier, Mme Fischer Böel a favorisé des changements qui, compte tenu des prémisses et des propositions initiales, peuvent apparaître assez satisfaisants. Par ailleurs, il fallait vaincre des résistances très aguerries. On peut donc en déduire que le premier objectif déclaré du Bilan de santé, selon lequel il s'agissait « d'un ajustement des réformes de 2003 », a été plus ou moins atteint, mais au détriment d'une analyse plus fine du fonctionnement du second pilier, pour qui de nouvelles tâches ont été confiées, sans grande lisibilité des ressources et sans une révision plus appropriée des procédures de fonctionnement.

\section{Le Bilan de santé apporte-t-il une réponse pour le futur de la PAC?}

L'objectif déclaré par Mme Fischer Böel était aussi d'apporter « une contribution au débat sur les priorités à venir dans le domaine de l'agriculture ». Dans ce cas, il faut s'interroger sur le positionnement de la conclusion du Bilan de santé dans les discussions de l'après 2013. En d'autres termes, est-ce qu'une proposition convaincante et durable émerge ? La retrouve-t-on dans les 
échanges qui ont eu lieu au sein du monde agricole ? Et cette proposition peut-elle être présentée à la table des négociations sur le réexamen du budget communautaire et sur les perspectives financières de la prochaine programmation?

En effet, lors de la conclusion du Bilan de santé, deux autres rendez-vous européens fondamentaux avaient eu lieu :

- en octobre 2008 à Limassol (Chypre) se tenait la conférence «Europe's rural areas in action: facing the challenges of tomorrow ", troisième grande rencontre sur la politique de développement rural, après celles de Cork en 1996 et de Salzbourg en 2003 ;

- en novembre 2008 à Bruxelles, la conférence "Reforming the budget, changing Europe », concluait la consultation publique sur le réexamen du budget et sa relance, l'ensemble devant se terminer en 2009.

Au cours de ces deux rencontres, deux sujets importants émergeaient : le premier concernait le rapport entre la PAC et les futures stratégies de l'UE; le second s'attachait au rapport entre le monde agricole et les représentations des autres intérêts en Europe.

\section{Les spécificités pour une Pac durable après 2013}

Le premier argument est qu'au sein de l'Union européenne, une confrontation complexe s'installe, due essentiellement à une nouvelle définition des fonctions mêmes de l'Union. En effet, après son élargissement à l'Est, d'abord les « non » français et hollandais à la Constitution, ensuite les difficultés de l'entrée en vigueur du traité de Lisbonne, enfin, les tentatives récurrentes de certains États membres de se détacher de l'Union et de diminuer leur contribution au budget européen, puis les effets de la crise économique, font que l'UE s'interroge sur ses objectifs pour les années à venir.
Le principal risque est la dissolution, sinon la défaite, du projet politique européen et, avec lui, la disparition du rôle de l'Europe dans un monde ouvert et multipolaire. Les contributions au débat sont nombreuses et, en général, de grande importance; l'éventail des positions est très large. Mais on peut identifier trois tendances stratégiques principales rencontrant une large convergence de vue :

- compétitivité, recherche, innovation :

l'UE doit promouvoir une croissance significative du soutien aux activités de recherche et à l'innovation, en faisant converger toutes ses politiques vers la compétitivité (en suivant les principes de Lisbonne) ;

- environnement et changements climatiques : il faut canaliser une plus grande partie des ressources dans cette direction, promouvoir les activités de recherche et de développement pour soutenir des objectifs attachés à l'environnement, aligner toutes les politiques à la durabilité (en suivant les principes de Göteborg) ;

- énergie : l'UE doit accroître sa sécurité énergétique, concentrer la recherche et les investissements sur l'efficience énergétique et le développement des énergies durables.

Ces choix impliquent une réforme profonde du budget qui pèsera sur les équilibres actuels et, en premier lieu, sur la politique agricole et la politique de cohésion qui, à ce jour, représentent 78,5\% du budget communautaire. La question n'est pas de savoir si l'engagement financier de l'UE pour l'agriculture va diminuer, mais de combien il va diminuer. Ce n'est pas un hasard si, parmi les résultats de la consultation publique voulue par la direction générale du budget (DGB) dans le réexamen du budget, la politique de la PAC a soulevé le plus grand nombre d'observations. Si l'on en résume les contenus, ces observations visent à demander, dans un premier temps : 
- la diminution des frais et, par la suite, le transfert de fonds du premier au second pilier,

- la baisse (sinon la suppression) des droits à paiement unique sinon, en tout cas, leur nivellement dans toute l'Union,

- le cofinancement pour le premier pilier.

Dans ce contexte, cependant, plusieurs interventions ont mis en évidence le fait que l'agriculture doit être considérée comme un secteur stratégique, qu'il faut moderniser et rendre compétitif ; on a souligné que la politique du développement rural est nécessaire pour affronter les «nouveaux défis » des changements climatiques, de la sécurité alimentaire (autant comme «food safety » que comme «food security »), de la biodiversité et de la sauvegarde des ressources naturelles. Tout cela souligne l'importance d'aligner la PAC aux nouveaux objectifs de l'UE :

- en explicitant précisément ce que les citoyens paient et pourquoi ils le paient dans le soutien à l'agriculture ;

- en s'orientant vers des politiques contractualisées dans lesquelles sont indiqués les engagements de l'exploitant agricole, payés avec les ressources publiques ;

- en proportionnant le montant des paiements des biens publics à l'augmentation des coûts ou à la diminution des revenus des exploitants agricoles.

Cela implique de nouveaux éléments tels que : le renforcement du second pilier à des rythmes bien plus rapides ; l'orientation de l'intervention publique vers les « nouveaux défis »; l'attention à la compétitivité et à l'innovation; l'intégration de la PAC dans le contexte plus complexe de la programmation communautaire, nationale et régionale.

En ce qui concerne le premier pilier et le régime de paiement unique (RPU), qui en représente la substance et qui utilise $70 \%$ de la totalité des frais de la PAC, il faut prendre en compte que son rôle d'instru- ment «d'assistance transitoire au changement» (Buckwell, 1997) ${ }^{4}$ est en train de s'épuiser et que les tentatives de le défendre comme un paiement de la «valeur d'existence » de l'agriculture risquent d'être faibles et complètement isolées dans le domaine européen. La survie de l'agriculture, dans certains territoires, est déjà largement garantie par les convenances du marché (en tout cas il faut travailler pour en améliorer la compétitivité). De même, en d'autres cas, par exemple dans les régions défavorisées ou les régions à haute sensibilité environnementale, le droit actuel à paiement unique n'est pas toujours suffisant pour stopper la diffusion de mauvaises pratiques agricoles, le déclin ou l'abandon (avec ce que cela comporte comme conséquences très lourdes sur l'environnement et ce que cela entraîne comme risques hydrogéologiques, etc.).

D'ailleurs, comme chacun le sait, un soutien lié à la terre et qui n'est pas considéré comme étant équivalent à de futurs engagements se traduit, en grande partie, par de plus grandes valeurs foncières et des coûts d'utilisation de la terre plus élevés, rendant l'activité agricole plus difficile et une compétitivité plus ardue à réaliser. Cette réflexion est également valable dans l'hypothèse extrême d'une régionalisation du paiement unique par hectare sur tout le territoire de l'UE, comme cela a été proposé. À plus forte raison, cette observation demeure valable pour les paiements effectués par le passé, légalisant, au moins jusqu'en 2013, la distribution inégale du soutien entre les exploitations et les territoires sur la période 2000-2002.

Dans le futur, le RPU sera dépassé, certes graduellement mais assez rapidement. Ce qui en restera, après 2013, sera essentiellement dû à l'inertie (à la " path dependency », comme diraient les économistes). Cette

4. Buckwell A. et al. (1997). Towards a Common Agricultural and Rural Policy for Europe en European Economy. European Commission, DG II, ${ }^{\circ} 5$. 
forme de paiement isole l'agriculture du débat sur l'avenir de l'UE et rend plus faible le projet d'une nouvelle PAC, modifiée, réformée et durable. Une défense à outrance du RPU pourrait, peut-être, permettre d'économiser quelques euros de plus dans le budget de la PAC, mais la dépense mal utilisée et mal distribuée ne peut pas engendrer une bonne politique agricole ni servir les intérêts de l'agriculture.

Cette analyse illustre l'absence, au Bilan de santé, de la contribution promise «... sur les priorités à venir dans le domaine de l'agriculture ». La réforme Fischler est à présent achevée. Mais quelles sont les propositions pour le futur :

- de miser encore sur le RPU totalement financé par Bruxelles après 2013 ?

- d'attendre encore cinq ans pour faire un autre petit pas dans la modulation ou pour relancer la régionalisation ou le rapprochement?

- ou quelle autre incitation?

Le risque est que la véritable réforme de la PAC soit conditionnée en raison des réductions économiques des futures perspectives financières.

\section{L'isolement de l'agriculture}

Le deuxième argument qui ressort de la confrontation entre le Bilan de santé et des deux rendez-vous de Chypre ${ }^{5}$ et de Bruxelles $^{6}$ concerne les institutions et la variété des participants. D'une part, s'agissant de l'approche sectorielle, les mandataires des intérêts du monde agricole et les représentants sectoriels étaient présents et ont participé aux débats sur le Bilan de santé, tandis que les intervenants des autres secteurs ont été peu nombreux ; d'autre part, une importante délégation était présente aux deux rendez-vous, alors que le monde agricole était quasiment absent.

5. Politique de développement rural (octobre 2008).

6. Réexamen du budget (novembre 2008).
Le Bilan de santé a essentiellement attiré les représentants du monde agricole et généré une confrontation interne et sectorielle. Les discussions se sont centrées sur des détails, peut-être très importants pour les lobbies respectives, mais finalement peu consistantes du point de vue économique et, plus encore, stratégique. Pour trouver un compromis final, on retouchait des détails comme le prélèvement additionnel pour les paiements supérieurs à 300000 euros, opération qui ne concerne que $0,04 \%$ des exploitations agricoles européennes. En même temps, la contribution de l'agriculture aux autres tables de discussion était insuffisante ou totalement absente.

Le cas de la conférence de Chypre a été exemplaire. Une participation massive s'est mobilisée, démontrant pourquoi la thématique du développement rural intéresse un ensemble diversifié d'institutions et de nombreux groupes professionnels :

- d'abord les institutions régionales et locales qui furent, pour la plupart, contournées par les dispositions du premier pilier (les droits à paiement unique transitaient directement, grâce aux organismes payeurs de Bruxelles aux bénéficiaires). Elles sont les protagonistes fondamentaux du second pilier ;

- puis les organisations territoriales et locales nées des expériences de «governance » introduites par l'initiative communautaire Leader et par différentes instigations nationales de programmation négociée (GAL, parcs naturels, districts ruraux ou agroalimentaires, etc.) ;

- ensuite les organisations sociales environnementalistes, mais aussi syndicales et toutes les associations intéressées par la préservation des valeurs culturelles, œnogastronomiques, historiques des régions rurales ou par la valorisation de la typicité des produits ;

- enfin le monde de la recherche et de l'Université. 
Par ailleurs, il est regrettable de constater comment, avec une rapidité exceptionnelle, le Copa/Cogeca ${ }^{7}$ a réagi avec un «non » péremptoire aux premières propositions de modulation obligatoire destinées à financer les défis du second pilier. Comme si les fonds transférés du premier au second pilier étaient perdus pour l'agriculture, bien que le cofinancement en double la valeur. Cette position de refus a été confirmée à nouveau à la fin du Bilan de santé : "L'accord met en danger le revenu des agriculteurs » (communiqué conjoint Copa/Cogeca). Ainsi, la représentativité du monde agricole fut marginale même lors de la «Consultation publique » sur le réexamen du budget, lancée en septembre 2007. La participation y fut importante : plus de 300 contributions ont été comptabilisées, provenant d'un large éventail d'institutions et de groupes professionnels ${ }^{8}$. Dans le cadre de la défense des intérêts des consommateurs et de protection de l'environnement, le groupe Copa/Cogeca a réaffirmé, dans un texte somme toute prévisible, la validité des objectifs originels de la PAC formulés dans le traité de Rome, se concluant par «Any cuts to the CAP budget will endanger these objectives ». Mise à part cette intervention, on ne compte que les contributions de la European Landowner Organisation (ELO) et seulement cinq autres communications du monde agricole (Grande-Bretagne, Danemark, Finlande et Allemagne).

Pour le bien de l'agriculture européenne, il est évident qu'il faut maintenant relancer rapidement et de manière forte la présence agricole à la table du réexamen du budget et qu'il est urgent de mettre en place une plus grande participation de l'agriculture à la réflexion sur le futur de la PAC toute

7. Comité des organisations professionnelles agricoles de l'Union européenne et Confédération générale des coopératives agricoles de l'Union européenne.

8. http://ec.europa.eu/budget/reform/issues/issues fr.htm entière, en particulier en ce qui concerne le second pilier. Ce passage ne sera peut-être pas sans douleurs, en considérant les nombreuses et très fortes résistances internes à toute modification, même marginale, de la PAC, mais cela est absolument nécessaire. La déconnexion de l'agriculture manifestée jusque-là est nuisible, elle produit l'isolement, elle mine les alliances et fait sauter des rendez-vous cruciaux pour le futur du secteur. Mais surtout, elle pénalise en son sein les meilleures forces entrepreneuriales, le changement générationnel, la mise en valeur des patrimoines alimentaires de qualité et, finalement, tout le développement d'un système agricole et alimentaire pouvant faire face à la compétition internationale.

\section{Un appel à la discussion}

Ce débat se veut l'interprète du rôle central que l'agriculture exerce et peut exercer en Europe. En même temps, il n'omet pas de rappeler le rôle fondamental que la PAC a exercé dans l'édification de l'Europe. Mais il ne faudrait pas que ce rôle primordial soit perdu. La faiblesse de la PAC frappe désormais les deux piliers :

- le premier en raison de l'ambiguïté de sa nature économique et de la difficile justification politique des droits à paiement unique ;

- le second par l'absence d'un dessein stratégique clair et du soin scrupuleux apporté dans la finalité de l'administration des ressources.

En Europe, nombreux sont ceux qui, depuis longtemps et avec réalisme, ont conscience de l'impérieuse nécessité de nouvelles solutions (Buckwell-Sotte, 1997) ${ }^{9}$. Des propositions récentes pour la $\mathrm{PAC}$ après 2013 ont émergé et ne manquent pas

9. Buckwell A., Sotte F. (1997) (Ed). Coltivare l'Europa, per une nuova politica e rurale comune. Liocorno Editori. 
(Bureau et Mahé 2008a ; Bureau et Mahé 2008b) ${ }^{10}$.

Lors du Conseil informel de $\mathrm{Brno}^{11}$, qui a eu lieu du 31 mai au 2 juin 2009, les ministres de l'Agriculture se sont interrogés sur trois domaines en particulier :

- le rôle des droits à paiement unique après 2013,

- leur connexion avec les mesures de marché et les mesures de développement rural, - la question des différents niveaux de paiement entre les États membres après 2013.

10. Bureau J.-C., Mahé L.-P. (2008a). CAP reform beyond 2013: an idea for a longer view, Projet de recherche. La PAC après 2013 ; www.notreeurope.eu/fr/axes/competition-cooperation-solidarite/projets/projet/la-pac-apres-2013/

Bureau J.-C., Mahé L.-P. (2008b). PAC : encore un effort? Telos, 04 juin 2008, www.telos-u.com/fr/ article/pac encore un effort

11. République Tchèque.
Encore une fois le débat s'est donc concentré sur le futur du premier pilier (Pupo D'Andrea, 2009) $^{12}$. À notre avis, au contraire, il est nécessaire et urgent de reprendre une réflexion collective sur le futur de toute la politique agricole. Il faut se concentrer d'abord sur la définition des objectifs et seulement après sur la définition des instruments, pour arriver à une corrélation claire entre les instruments et les objectifs. Cette approche participerait avec succès à l'examen des intérêts non agricoles lors des discussions sur le budget et sur les futures perspectives financières de la Communauté européenne.

La PAC après 2013 mérite que l'on élargisse et que l'on approfondisse le débat. À travers ce type d'expression, nous espérons avoir réussi cet objectif au profit des lecteurs d'Économie rurale.

12. Pupo D'Andrea M.-R. (2009). Finestra sulla $P A C$. Agriregionieuropa, n.17, juin.

\section{BIBLIOGRAPHIE COMPLÉMENTAIRE}

De Filippis F. (Ed.) (2008). Il futuro della PAC dopo l'Health Check. Quaderni Gruppo 2013, Rome, Edizioni Tellus. Commission des Communautés européennes (2007). Réformer le budget, changer l'Europe : document de consultation publique en vue du réexamen $d u$ budget 2008/2009. Bruxelles, 12 septembre 2007, SEC(2007) 1188 final.

Fischer Böel M. (2006). The European Model of Agriculture. Oulu, Finlande, septembre.
Sotte F. (2005). From CAP to Carpe: The State of the Question. In Ortner K.M. (Ed.), "Assessing rural development policies of the Common Agricultural Policy", Wissenschaftsverlag, Vauk, Kiel, Introductory Paper to the $87^{\circ}$ EAAE Seminar, Wien, April.

Sotte F. (2008). La nature économique des Droits à paiement unique. SFER, Paris, Économie Rurale, $\mathrm{n}^{\circ}$ 300, p. 65-70. 